\title{
Predictors of Happiness in Adults with Disability: Comparison Depending on Poverty Level and Gender
}

\author{
Sikung Nho, Junyoung Jeong, Miok Kim¹ \\ Jeonbuk National University, Department of Social Welfare
}

\begin{abstract}
This study explored the differences in the happiness of adults with disability depending on poverty level and gender. It also identified the factors that affected the happiness of each group. To this end, this study used the third survey(2018) data from the Second-Wave Panel Survey of Employment for the Disabled and analyzed data on 3,778 disabled people aged 20 or older. Groups were classified into four groups of no-poverty males with disability, nopoverty females with disability, males with disability suffering poverty, and females with disability suffering poverty. The results showed that the health status, interpersonal relationships, self-esteem, social participation, and happiness of no-poverty people with disability were higher than people with disability suffering poverty, and experiences of discrimination were observed to be higher in people with disability suffering poverty than in no-poverty people with disability. Also, even between no-poverty groups, health status and social participation were higher in no-poverty males with disability than in no-poverty females with disability. The results on the happiness predictors showed that health status, socioeconomic status, satisfaction with residence, and self-esteem had positive effects on the happiness of all groups. Whether interpersonal relationships and a person had a chronic disease had significant effect only in the group of no-poverty people with disability, while experiences of discrimination were found to have a negative effect only in the group of females with disability. Based on these results, this study provided implications regarding economic-related issues in adults with disability and the necessity of different approaches for each group.
\end{abstract}

Keywords: economic inequality; experiences of discrimination; gender gap; health; i ntergroup comparison

\footnotetext{
1 Corresponding author
} 